\title{
Estructuración y función del miocardio ventricular canino (Canis lupus familiaris): banda miocárdica ventricular
}

Rodrigo De Lavalle*, Esp. ${ }_{1}$, Luis Rendón, $\mathrm{MVZ}_{2}$

Clínica Veterinaria Mastervet, Barranquilla, Colombia

${ }_{2}$ Clínica Veterinaria Layca, Soledad, Atlántico, Colombia

Recibido: 11 de diciembre del 2014 Aprobado: 9 de abril del 2015

*Autor de correspondencia: Rodrigo De Lavalle. Barrio San Vicente, calle 86 n. ${ }^{\circ}$ 47-78. Barranquilla, Colombia. Teléfono: (57) 6 3738698. Correo electrónico: rodrigodelavalle@gmail.com

Cómo citar este artículo: De Lavalle R, Rendón L. Estructuración y función del miocardio ventricular canino (Canis lupus familiaris): banda miocárdica ventricular. Spei Domus. 2015;11(22):25-35. doi: http://dx.doi.org/10.16925/sp.v11i22.1156

Resumen. Introducción: actualmente, hay nuevas herramientas de diagnóstico y terapias para enfermedades cardiacas, pero sin consenso científico serio sobre los interrogantes acerca de la estructura normal del corazón y su función. La banda helicoidal miocárdica ventricular de Torrent-Guasp es el nuevo y revolucionario concepto en la comprensión global, en tres dimensiones de la arquitectura funcional del miocardio ventricular. Este concepto define la arquitectura de tejidos y fuerzas netas desarrollada en la masa ventricular. Materiales y métodos: se utilizaron 36 corazones de caninos de diferentes pesos, razas y sexos, a fin de demostrar la existencia de la banda miocárdica ventricular en el miocardio canino mediante una disección de tipo manual. Resultados: la disección realizada mostró una gran tendencia hacia una estructura helicoidal continua, con una disposición longitudinal en las fibras miocárdicas ventriculares, formando una única banda muscular funcional que empieza a la altura de la arteria pulmonar y termina a nivel de la aorta. Se identificaron los diferentes segmentos de la banda: el derecho (porcentaje de presentación $100 \%)$, el izquierdo (100\%) respecto a las partes de la lazada basal; y en la disección de la lazada apexiana: el descendente (100\%) y el ascendente (100\%). Conclusiones: se demostró la presentación de la banda miocárdica ventricular en el miocardio canino (Canis lupus familiaris) y se identificaron sus estructuras. Este es un nuevo concepto de la anatomía del miocardio ventricular canino, donde se evidencia macroscópicamente la entramada red de la doble helicoide. Según la teoría de Torrent-Guasp, el miocardio canino tendría la siguiente secuencia de contracción: contracción de la lazada basal, contracción del segmento descendente y ascendente de la lasada apexiana, y la expulsión y succión ventricular.

Palabras clave: ascendente, descendente, diástole, helicoide, sístole. 


\title{
Structure and function of the canine ventricular myocardium (Canis lupus familiaris): Ventricular myocardial band
}

\begin{abstract}
Introduction: currently, there are new diagnostic tools and therapies for heart disease, but with no serious scientific consensus on the questions about the normal structure and function of the heart. Helical ventricular myocardial band of Torrent-Guasp is the revolutionary new concept in understanding global, three-dimensional, functional architecture of the ventricular myocardium. This concept defines the architecture and net forces developed within the ventricular mass. Materials and methods: 36 hearts of dogs of various weights, breeds and sexes were used to demonstrate the existence of a ventricular myocardial band in the canine myocardium by manual dissection. Results: the dissection performed showed a strong trend towards a continuous helical structure with a longitudinal arrangement of ventricular myocardial fibers, forming a single functional muscular band that begins at the height of the pulmonary artery and ends at the level of the aorta. Different segments of the band were identified: right (presence percentage $100 \%)$ and left (100\%) with respect to parts of the basal loop, and descendant (100\%) and ascendant (100\%) in the dissection of the apical loop. Conclusions: the presence of the ventricular myocardial band in the canine myocardium (Canis lupus familiaris) was demonstrated and their structures identified. This is a new concept in the anatomy of the canine ventricular myocardium, where the complex network of the double helicoid is macroscopically evidenced. According to the theory of Torrent-Guasp, the canine myocardium would have the following contraction sequence: contraction of the basal loop, contraction of the descendant and ascendant segments of the apical loops, and ventricular ejection and suction.
\end{abstract}

Keywords: ascendant, descendant, diastole, helicoid, systole.

\section{Estruturação e função do miocárdio ventricular canino (Canis lupus familiaris): banda miocárdica ventricular}

Resumo. Introdução: atualmente, há novas ferramentas de diagnóstico e terapias para doenças cardíacas, mas sem consenso científico sério sobre as questões referentes à estrutura normal do coração e sua função. A banda helicoidal miocárdica ventricular de TorrentGuasp é o novo e revolucionário conceito na compreensão global em três dimensões da arquitetura funcional do miocárdio ventricular. Esse conceito define a arquitetura de tecidos e forças resultantes desenvolvida na massa ventricular. Materiais e métodos: foram utilizados 36 corações de caninos de diferentes pesos, raças e sexos a fim de demostrar a existência da banda miocárdica ventricular no miocárdio canino mediante uma dissecção de tipo manual. Resultados: a dissecção realizada mostrou uma grande tendência a uma estrutura helicoidal contínua, com uma disposição longitudinal nas fibras miocárdicas ventriculares, que formam uma única banda muscular funcional que começa na altura da artéria pulmonar e termina no nível da aorta. Foram identificados diferentes segmentos da banda: o direito (porcentagem de apresentação $100 \%$ ), o esquerdo (100\%) a respeito das partes da laço basal; na dissecção do laço apical: o descendente (100\%) e o ascendente (100\%). Conclusões: demonstrou-se a apresentação da banda miocárdica ventricular no miocárdio canino (Canis lupus familiaris) e foram identificadas suas estruturas. Este é um novo conceito da anatomia do miocárdio ventricular canino, onde se evidencia macroscopicamente a entrelaçada rede da dupla hélice. Segundo a teoria de Torrent-Guasp, o miocárdio canino teria a seguinte sequência de contração: contração da laço basal, contração do segmento descendente e ascendente da laço apical, e a expulsão e sucção ventricular.

Palavras-chave: ascendente, descendente, diástole, hélice, sístole. 


\section{Introducción}

El corazón se define como el órgano principal del aparato circulatorio. Es un músculo estriado hueco que actúa como una bomba aspirante e impelente, que aspira hacia las aurículas la sangre que circula por las venas y la impulsa desde los ventrículos hacia las arterias. El miocardio ventricular en los caninos ha sido descrito por Brudras et al. como un músculo que está compuesto por tres capas (interna, media y externa), que se mezclan entre sí formando un espiral en el ápice del corazón [1].

Sisson y Grossman describen el corazón como dos cavidades con fibras superficiales que se dirigen desde la base hacia el ápex, luego vuelven sobre sí mismas en forma de espiral hacia el vértice y se dirigen profundamente hacia arriba, hasta terminar en los músculos papilares formando lazos en remolino. Anotan, además, que estas fibras profundas parecían propias de cada ventrículo pero comunes a las dos, encorvándose alrededor de la pared correspondiente, pasando por el tabique al lado opuesto y encorvándose nuevamente en el lado opuesto [2].

Otra descripción es la de Randall et al., que describe el corazón constituido por cuatro cámaras: dos aurículas y dos ventrículos que provocan la expulsión de sangre hacia el interior del sistema circulatorio por dos mecanismos: una contracción (sístole) y una dilatación (diástole). La fase diastólica de los ventrículos es ayudada por una contracción auricular para terminar el llenado de los ventrículos [3]. Y por último, Cunningham et al. nos describen que la sístole tiene lugar en dos fases: primero se contraen las aurículas y luego lo hacen los ventrículos [4].

En efecto, el miocardio ventricular ha demostrado ser notablemente resistente a los análisis macroscópicos de la anatomía funcional, hasta que en 1954 el científico español Torrent-Guasp señalara, en un trabajo teórico, la imposibilidad de atribuir a la contracción auricular el llenado de las cavidades ventriculares, con lo que abogó por la existencia de una dilatación ventricular activa. Así, volvió a lo que preconizó el griego Eristrato hace 2300 años, apoyado por Galeno unos 430 años después, hasta que en el siglo Xvir Harvey afirmara lo contrario: que su principal acción es la expulsión de sangre a las arterias, de forma tal que el llenado ventricular pasó a ser considerado secundario a la contracción auricular [5].

Las ideas de Torrent-Guasp fueron recogidas y confirmadas por Brecher en 1956, al demostrar experimentalmente la existencia de una succión ventricular diastólica [6]. La fuerza con que se lleva a cabo la succión ventricular solo podía ser atribuida a una acción muscular específica realizada por el miocardio ventricular [7]. Según trabajos desarrollados por el mismo Torrent-Guasp, apoyado en complejos estudios físico-matemáticos, el miocardio es una banda muscular continua que se inicia en la raíz de la arteria pulmonar y termina en la aorta, tras describir dos vueltas de espiral configurando un helicoide. Como entre función y estructura existe un estrecho paralelismo, la transmisión del estímulo para la contracción cardiaca debería transmitirse longitudinalmente a lo largo de la banda [8].

La banda miocárdica ventricular de TorrentGuasp es un nuevo concepto, que constituye un terreno importante y firme para la reconciliación de algunos conceptos en la medicina cardiovascular. Este concepto es deslumbrante por su singular e intrínseco ingenio y, por lo tanto, parece "excesivamente simple en principio pero maravillosamente complicado en los detalles" [9].

Se han determinado cuatro bases arquitecturales anatómicas del corazón, en las que la mitad apexiana del ventrículo izquierdo evidencia una trayectoria laminar en espiral desde el epicardio hacia el endocardio. En la mitad basal del ventrículo izquierdo, existe una uniformidad estructural -así como en la mitad apexiana- de trayectorias laminares circularmente imbricadas, expresadas solo en la pared libre del ventrículo izquierdo. En esta zona, es muy importante apreciar cómo las fibras musculares, pertenecientes a la pared libre del ventrículo izquierdo, pasan de afuera hacia adentro sin efectuar inserción alguna en el anillo mitral. En la mitad apexiana del ventrículo derecho, igual a lo que se encuentra en la parte apexiana del ventrículo izquierdo, se evidencia una trayectoria laminar en grupos sucesivos, y en la mitad basal derecha del ventrículo derecho hay la misma estructuración de la trayectoria laminar de la parte basal del ventrículo izquierdo [10].

Estos resultados evidencian la existencia de un estrecho paralelismo en la estructura de las cuatro regiones miocárdicas, todas ellas constituidas por una serie de trayectorias laminares circularmente imbricadas. Solo un modelo de cuerda adoptada en una forma helicoidal permite expresar coherentemente la relación existente entre los cuatro hechos anatómicos descritos y, al mismo tiempo, el porqué 
de la trayectoria opuesta, seguida por las fibras musculares en las regiones basales y en las apexianas. Con este modelo, podemos explicar la diferencia existente entre el grosor de las paredes libres de ambos ventrículos: en la constitución de la pared libre del ventrículo derecho solo interviene una lazada del helicoide, mientras que la pared libre del ventrículo izquierdo está compuesta por dos lazadas [9].

Con estos estudios, el plan general de organización del miocardio ventricular está representado por un conjunto de fibras retorcido sobre sí mismo como una cuerda y lateralmente aplastado a modo de banda, que describe dos vueltas de espiral y define un helicoide. Pero, evidentemente, en tal concepción faltaba algo: precisar dónde estaban ubicados ambos extremos de aquella banda muscular. Mediante el estudio de seres (gusanos, reptiles, peces, aves, mamíferos) que representan los sucesivos hitos de la línea filogenética conducente a los animales superiores actuales, fueron evidenciados hechos que mostraron claramente que el principio y el fin de la banda miocárdica ventricular debían ser encontrados, respectivamente, en la raíz de la arteria pulmonar y en la aorta [10].

Se les designaron nombres a diversas regiones del corazón, y se efectuó una división de la banda, basada en una serie de hechos anatómicos, mediante la cual son individualizados diversos segmentos, todos ellos con una propia y distintiva personalidad morfológica. Dada la configuración helicoidal adoptada por la banda miocárdica ventricular, pueden ser distinguidas dos lazadas: la basal y la apexiana. Estas dos lazadas quedan delimitadas por tres referencias anatómicas: la raíz de la arteria pulmonar, la dobladura de la banda y la raíz de la aorta. La lazada basal discurre desde la raíz de la arteria pulmonar a la dobladura central de la banda, mientras que la lazada apexiana queda definida por aquella dobladura y la raíz de la aorta, como se observa en el diagrama de Torrent-Guasp, en la figura 1 [5].

A su vez, cada lazada puede ser dividida en dos segmentos correspondientes a hechos anatómicos. La lazada basal se divide en: un lado derecho, constituido por la pared libre del ventrículo derecho, que limita por fuera con el orificio tricuspídeo y está dividido por la parte posterior de la cavidad derecha, para continuar con la parte izquierda, que conforma la pared libre del ventrículo izquierdo, definida por fuera del orificio mitral. La parte apexiana queda dividida por dos segmentos: uno descendente, constituido por las fibras que desde la dobladura de la banda descienden hacia el ápex, en donde retorna hacia la base configurando el segmento ascendente. Estas dos estructuras son separadas por el músculo papilar anterior, asumiendo el papel de frontera para separar ambos segmentos, el descendente y el ascendente, como podemos apreciar en la figura 1 [5].

Varios anatomistas veterinarios describen el corazón de manera diferente, sin llegar a establecer un concepto de forma, organización y función de

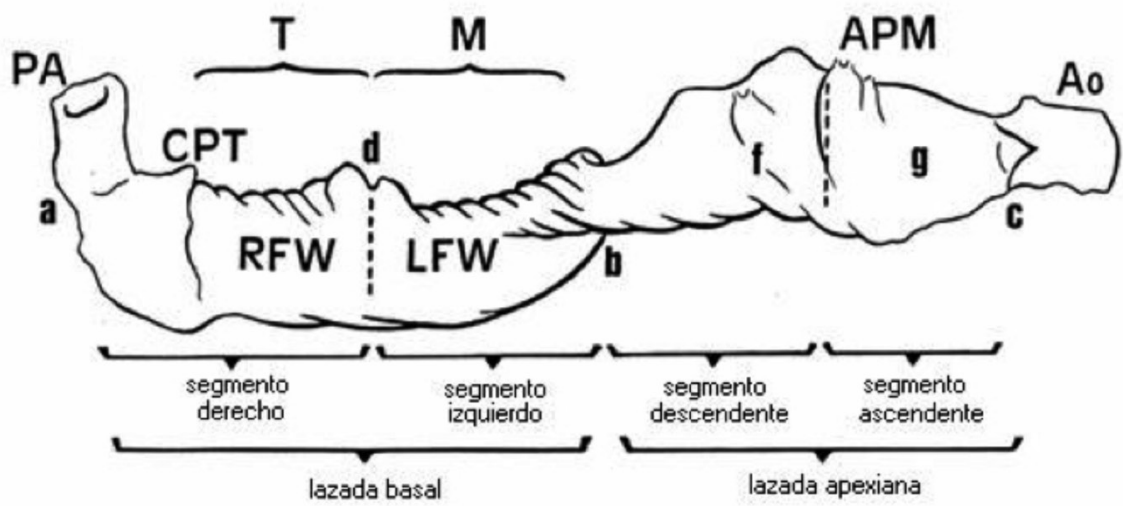

Figura 1. Reproducción de la banda miocárdica según Torrent-Guasp; PA: arteria pulmonar. Ao: aorta. T: borde lateral o externo del orificio tricúspide. RFW: pared libre del ventrículo derecho. M: borde lateral o externo del orificio mitral. LFW: pared libre del ventrículo izquierdo. СРT: cordón fibroso pulmotricuspídeo. APM: músculo papilar anterior. Raíz de la arteria pulmonar; a) surco interventricular posterior, d) (señalado con línea de puntos) dobladura de la banda, b) raíz de la aorta, c) segmento descendente, f) segmento ascendente, g) (tomado y modifica de TorrentGuasp)

Fuente: elaboración propia 
las fibras cardiacas. Torrent-Guasp descubrió que la parte ventricular del corazón era una banda con continuidad muscular que se replegaba sobre sí misma en forma de hélice, con lo cual se relaciona la anatomía y la fisiología, es decir, la forma y la función, apoyando la hipótesis de la presencia de una acción muscular activa que produce la succión durante la diástole ventricular.

El propósito principal es analizar objetivamente la presentación de la banda miocárdica ventricular y la de cada uno de los segmentos de cada lazada en el miocardio canino, junto con un intento de aclarar algunos aspectos, todavía no dilucidados, del funcionamiento de la banda miocárdica en la clínica diaria. Con la descripción de la banda, podemos explicar que cada segmento tiene una contracción activa, lo que lleva al llenado de los ventrículos y a la expulsión de sangre hacia las arterias. Hoy en día, hay muchas dudas acerca del funcionamiento y su correlación clínica en las diferentes ayudas diagnósticas, así como enfermedades que afectan a nuestras mascotas.

La integración de la anatomía y la función del miocardio ventricular son fundamentales para una completa comprensión de la fisiología cardiaca, lo que podría revelar conocimientos clave para futuros estudios experimentales y procedimientos clínicos [10].

\section{Materiales y métodos}

Se estudiaron 36 corazones caninos con pesos comprendidos entre 2 y $70 \mathrm{~kg}$, de ambos sexos, de diferentes razas y edades, obtenidos de individuos eutanasiados en los centros veterinarios Mastervet, Dogtorcat y Layca, ubicados en Barranquilla y Soledad (Atlántico, Colombia). Las disecciones de los corazones de los pacientes eutanasiados fueron realizadas respetando la legislación colombiana y estadounidense sobre la protección de animales usados en experimentación o sobre el empleo de agentes biológicos $\mathrm{u}$ organismos genéticamente modificados, establecidos en la Ley 84 de 1989.

Después de la eutanasia de cada espécimen, es retirada la muestra anatómica por una incisión lateral en el espacio intercostal. Luego se masajea y se lava con agua corriente para despejar los ventrículos de las aurículas y la grasa cardiaca; la aorta y la arteria pulmonar quedaron separadas, con los orificios auriculoventriculares y los músculos ventriculares. El paso a seguir fue colocar la muestra a hervir por veinte minutos y luego dejarla reposar al aire libre para su posterior manipulación.

A los corazones recolectados de los caninos eutanasiados se les practica una disección manual, y solo se utiliza como material una tijera de mayo recta para separar la arteria pulmonar y la aorta. La disección de toda la banda miocardiaca es manual.

En una memoria electrónica, se digitalizan y almacenan los datos, los registros correspondientes al hallazgo de cada segmento de la banda miocardiaca, la lazada basal que corresponde a un segmento derecho e izquierdo, y la lazada apexiana con sus segmentos descendentes y ascendentes.

Se analizó la presentación de cada segmento de la banda, en la que se tomaron los segmentos como variables, y se evaluó el porcentaje y la frecuencia relativa de presentación de las 36 muestras, y sus posibles variaciones entre los corazones estudiados. Se utilizó el paquete estadístico spss.

\section{Resultados}

La disección que se hizo a los diferentes corazones mostró una gran tendencia de una estructura helicoidal continua, con lo que evidenció una disposición longitudinal en las fibras miocárdicas ventriculares y formó una única banda muscular funcional que empieza a la altura de la arteria pulmonar y termina a nivel de la aorta. Este músculo envuelve el ventrículo derecho y parte del ventrículo izquierdo, conectando con una estructura helicoidal que se inicia en el anillo basal, va hacia el interior del ventrículo izquierdo y hacia el vértice cardiaco, y regresa para conectar con la aorta, envolviendo toda la anatomía del corazón.

Se identificaron los diferentes segmentos de la banda en las disecciones realizadas. Se identificó el segmento derecho con un porcentaje de presentación de $100 \%$; en el segmento izquierdo, la frecuencia de presentación fue de $100 \%$ con respecto a las partes de la lazada basal; en la disección de la lazada apexiana, se tuvo un grado de dificultad en la dobladura cardiaca, pero la frecuencia de presentación del segmento descendente fue de $100 \%$; el segmento ascendente, que sería el encargado de la diástole cardiaca y que termina en la arteria aorta, tuvo una frecuencia de presentación de $100 \%$.

La técnica de disección realizada en los miocardios ventriculares caninos se llevó a cabo de la siguiente forma: se inició con la separación de la 


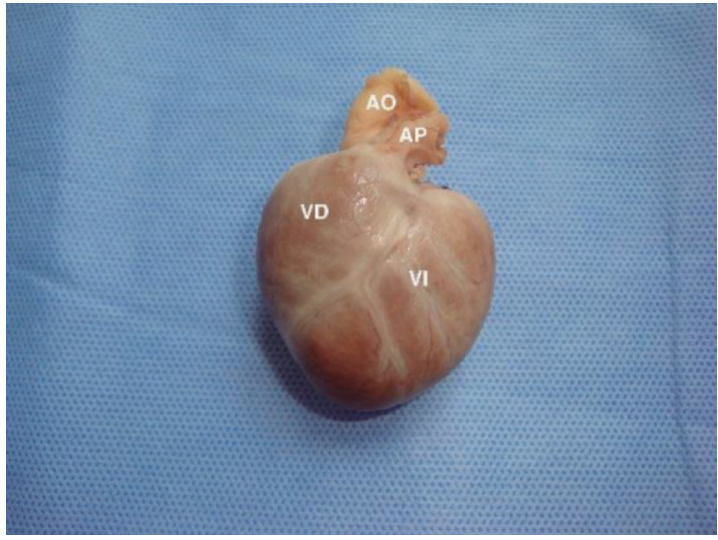

Figura 2. Vista de la superficie izquierda de un corazón canino recién procesado. Se despejaron los ventrículos de las aurículas y la grasa cardiaca, quedando la aorta y la arteria pulmonar separadas

Fuente: elaboración propia

arteria pulmonar y la aorta, (figuras 2 y 3 ). En la zona lateral del corazón ubicamos la zona interventricular que presentan algunas de las fibras superficiales (es decir, las fibras aberrantes); estas hacen un puente en el surco interventricular anterior, en el que se practicó una incisión manual de estas fibras, con el fin de mover a un lado el ventrículo derecho (figura 2). Así, se llega al borde posterior de la cavidad lineal del ventrículo derecho, representado

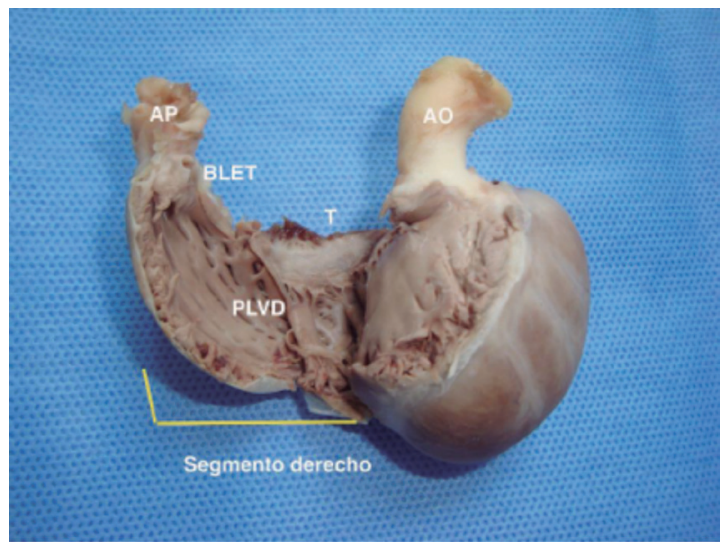

Figura 4. Imagen de la disección de la lazada basal mostrando el segmento derecho del corazón de un canino. AP: arteria pulmonar, AO: arteria aorta, PLVD: pared libre del ventrículo derecho, BLET: borde lateral externo del orificio tricúspide, T: orificio tricúspideo Fuente: elaboración propia

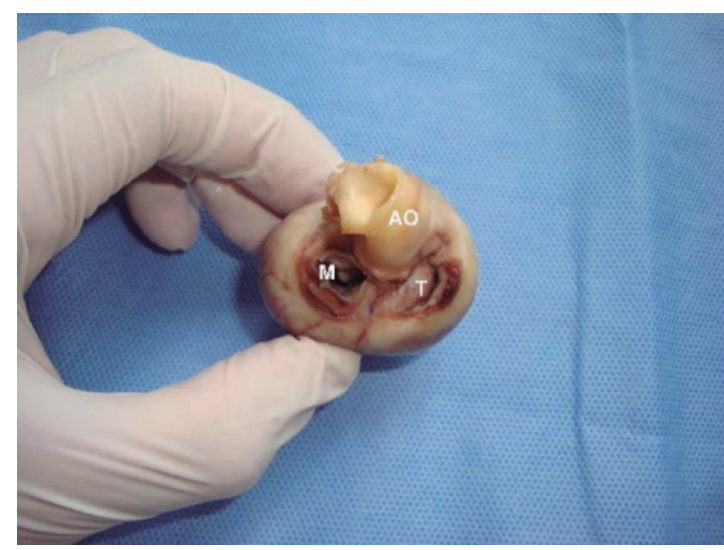

Figura 3. Vista basal de un corazón canino recién procesado. Se despejaron los ventrículos de las aurículas y la grasa cardiaca, quedando la aorta y la arteria pulmonar separadas. T: orificio tricúspide, $\mathrm{M}$ : orificio mitral

Fuente: elaboración propia

por la parte inferior lineal del ángulo diedro formado por la pared libre del ventrículo derecho y del septo interventricular (figura 4); en esta parte, tenemos el segmento derecho de la lazada basal de la banda miocárdica. El borde posterior de la cavidad lineal del ventrículo izquierdo es expuesto al empujar lateralmente la pared libre del ventrículo derecho. Tras la disección de estas fibras predominantes, podemos ver fácilmente que este camino

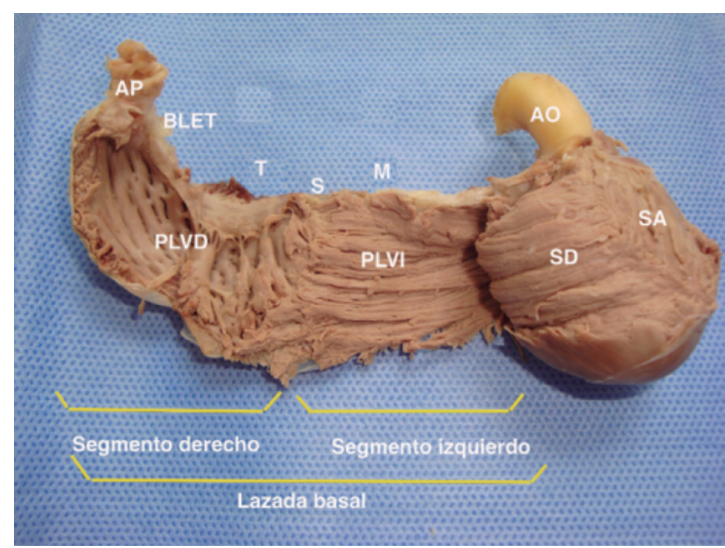

Figura 5. Imagen de los componentes de la lazada basal. Segmento derecho e izquierdo del corazón de un canino. AP: arteria pulmonar, AO: arteria aorta, PLVD: pared libre del ventrículo derecho, BLET: borde lateral externo del orificio tricúspide, PLvi: pared libre del ventrículo izquierdo, M: orificio mitral, T: orificio tricuspídeo, s: surco interventricular subsinusal, sD: segmento descendente, sA: segmento ascendente Fuente: elaboración propia 
rodea al ventrículo izquierdo hasta la raíz de la aorta (figura 5). En esta etapa, termina la disección del segundo componente de la lazada basal, el segmento izquierdo. Al reducir su anclaje con el trígono fibroso izquierdo, terminamos la disección de la lazada basal de la banda miocárdica ventricular (en este punto, es importante notar que algunas fibras pertenecientes al segmento descendente se hunden en el ventrículo izquierdo).

Para continuar con las disecciones, por inspección desde el lado del ventrículo izquierdo, podemos distinguir claramente dos estratos musculares (figura 5): primero, la capa profunda pertenece al segmento descendente, y segundo, el más superficial pertenece al segmento ascendente. Estas fibras forman un cruce en ángulo recto, también visible desde el lado del ventrículo derecho. La raíz de la aorta se encuentra adherida al trígono fibroso derecho, y para separar los estratos descritos, pasamos en medio de las fibras verticales (más superficial, el segmento ascendente) y las fibras horizontales (más profundo, el segmento descendente), haciendo una debridación manual de su anclaje a los trígonos fibrosos derechos.

Procedemos luego a la parte más delicada de la disección: el "desmontaje de la aorta". De tal manera, avanzando por la trayectoria de la fibra predominante,

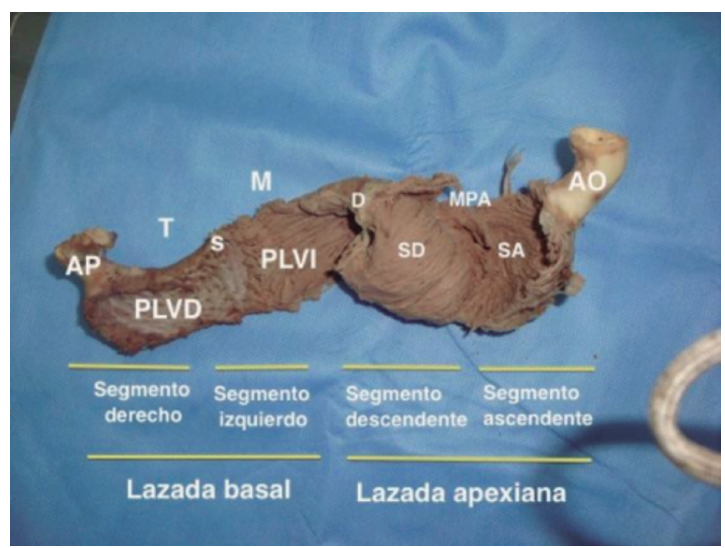

Figura 6. Imagen de los componentes de la lazada basal. Segmentos derecho e izquierdo del corazón de un canino. AP: arteria pulmonar, AO: arteria aorta, PLVD: pared libre del ventrículo derecho, BLET: borde lateral externo del orificio tricuspídeo, PLVI: pared libre del ventrículo izquierdo, M: orificio mitral, T: orificio tricuspídeo, s: surco interventricular subsinusal, SD: segmento descendente, SA: segmento ascendente, MPA: musculo papilar anterior, D: dobladura de la banda Fuente: elaboración propia separamos la aorta con las fibras pertenecientes al segmento ascendente del resto de la masa del ventrículo izquierdo, para finalmente llegar a la disección de la parte apexiana de la banda miocárdica ventricular (figura 6). Una simple rotación de $90^{\circ}$ alrededor del vértice desenreda los segmentos del bucle apical. La banda miocárdica ventricular de Torrent-Guasp aparece ahora con la arteria pulmonar y la aorta.

El modelo de cuerda helicoidal también muestra las relaciones transversales y bidimensionales, junto con la disposición anatómica de diferentes grosores de las paredes del ventrículo izquierdo y del derecho. La pared libre del ventrículo izquierdo, que se compone de dos bucles, es más gruesa que la pared libre del ventrículo derecho, que está constituido por un solo bucle (figuras 7 y 8 ).

A fin de mostrar los componentes de cada estructura, como la pared libre del ventrículo izquierdo, la del derecho y el séptum interventricular, después de extender la banda, se enrolla de nuevo hasta reconfigurar el corazón, y a continuación se hace un corte

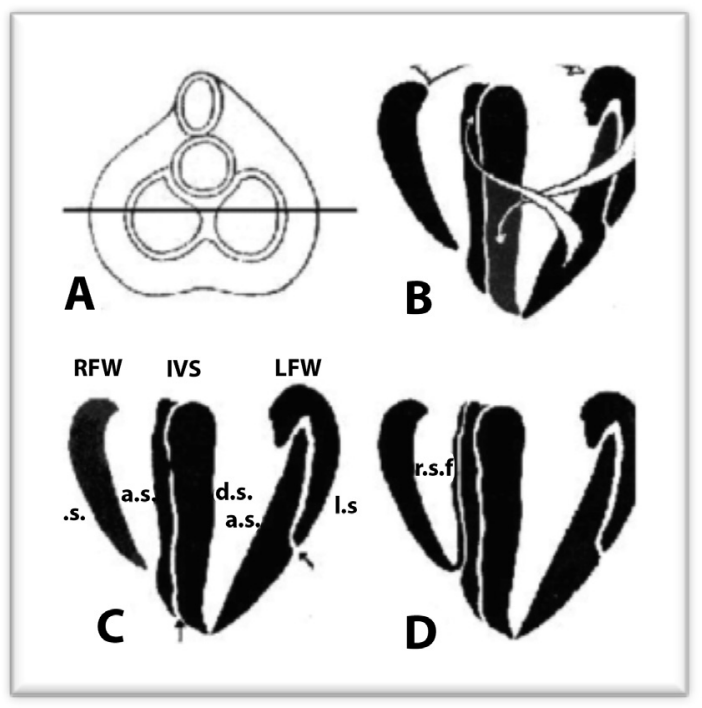

Figura 7. Imagen del esquema que indica el corte longitudinal, efectuado en la dirección base-ápex, que divide en dos mitades la masa ventricular. B: las flechas indican el trayecto sucesivamente descrito en el espacio por los segmentos de la banda, C RFW: pared ventricular derecha, IVs: tabique interventricular, LFW: pared libre del ventrículo izquierdo, r.s.: segmento derecho, a.s.: segmento ascendente, d.s.: segmento descendente, l.s.: segmento izquierdo, D: señala las fibras que provienen del ventrículo derecho Fuente: adaptado de Torrent-Guasp 
longitudinal desde la base hasta el ápex (figuras 7 y 8 ). Así, la masa ventricular queda dividida en dos mitades: el segmento derecho está constituido por la lazada basal (único componente de la pared libre del ventrículo derecho) y el segmento izquierdo (parcial componente de la pared libre del ventrículo izquierdo), y abraza la lazada apexiana, el segmento ascendente (el otro componente parcial de la pared libre del ventrículo izquierdo) y el descendente. A su vez, el tabique interventricular está conformado por el cruce del segmento descendente con el ascendente (figura 8).

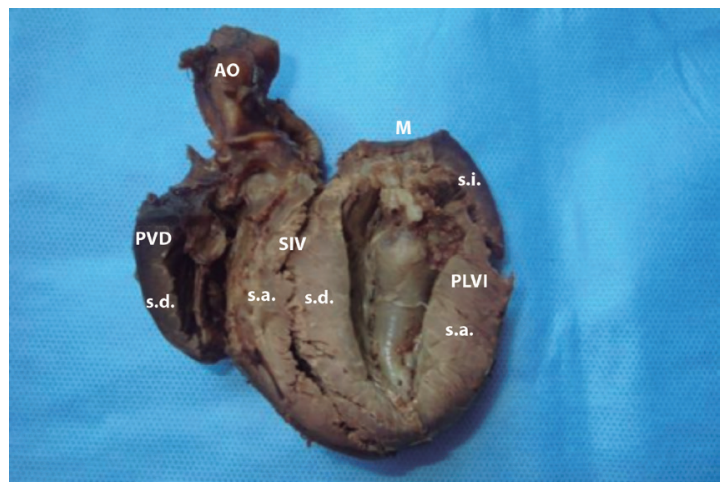

Figura 8. Imagen de un corazón canino cortado desde la base hacia el ápex, luego de haber diseccionado la banda miocárdica, en la que se observa la conformación interna del miocardio séptum. PVD: pared ventricular derecha, AO: arteria aorta, M: orificio mitral, PLVI: pared libre del ventrículo izquierdo, siv: tabique interventricular, s.d.: segmento derecho, s.a.: segmento ascendente, s.I.: segmento izquierdo.

Fuente: elaboración propia

\section{Discusión}

Varios anatomistas veterinarios como Brudras et al., Sisson y Grossman han estudiado la estructura del corazón describiendo los ventrículos y las fibras musculares de diferentes maneras [2]; Andrés Vesalio (siglo xvi) explica que las fibras adoptan la misma disposición que los mimbres de una cesta; Richard Lower (siglo XVII) describe por primera vez la disposición de las fibras del corazón en varias capas enrolladas en espiral; Jean-Baptiste de Sénac dedica muchas páginas a la disposición de las fibras cardiacas [11]; Thomas Bartholin (siglo xvII) y Jules Germain Cloquet (siglo XIX) hacen una descripción muy detallada de las fibras cardiacas; y Robert Koch (siglo XIX) muestra cortes histopatológicos con la disposición de las fibras cardiacas [12].
Está muy establecida la presencia de las cuatro cavidades (dos aurículas y dos ventrículos) y la disposición de las fibras musculares entrecruzadas en varios niveles, así como los modelos teóricos, más o menos acertados, del mecanismo por el cual la sangre circula por el interior del corazón. Pero poco se conocía con certeza acerca de la estructura anatómica mayor del corazón y de la disposición de las fibras musculares [13]. Con nuestro estudio, pudimos demostrar la banda miocárdica de TorrentGuasp en el miocardio canino, que se presenta en el espacio y en el tiempo como una doble hélice. Se corrobora con Poveda et al. que, a través de una nueva técnica de diagnóstico por imagen de resonancia magnética por tensor de difusión, se probó la existencia de una conectividad inequívoca entre las fibras ventriculares, dispuesta de manera continua en una doble hélice que conforman ambos ventrículos en cuatro corazones caninos [10].

El pensamiento establecido hasta la fecha acerca del movimiento del corazón (sístole-diástole) se creía activo-pasivo, en el sentido de que la primera se producía por la acción de contracción muscular y la segunda, por relajación de la musculatura cardiaca [14]. Con la identificación de la banda miocárdica ventricular y cada uno de sus componentes, se apoya la teoría de que la acción muscular produce la succión durante la diástole ventricular. Así, la contracción del segmento ascendente de la banda miocárdica provoca, paradójicamente, el aumento del volumen del ventrículo.

El conocimiento clásico de la mecánica cardiaca de que los movimientos transversales causan el estrechamiento y el ensanchamiento, y la constricción y la dilatación de las cavidades ventriculares, es considerado de mayor relevancia cuantitativa. Ha quedado inculcado en la mente de todos que el corazón se constriñe y se dilata, más que se acorta y se alarga, producto de los movimientos longitudinales a causa del acortamiento y el alargamiento de las cavidades ventriculares. Con aquella idea de la mecánica cardiaca, basada en la constricción y la dilatación ventricular, puede ser fácilmente explicada la expulsión de sangre, pero no la succión de sangre consecuencia de la dilatación de los ventrículos [15].

Con la presencia de la banda helicoidal en el miocardio canino, podemos explicar el llenado de los ventrículos, dado que la anatomía está relacionada con la fisiología de la contracción. La contracción agonista de su segmento descendente origina, 
mediante el acercamiento de la base al ápex, una disminución de volumen ventricular a causa de la expulsión de la sangre a las grandes arterias (pulmonar y aorta), mientras la contracción antagonista de su segmento ascendente origina, mediante el alejamiento de la base del ápex, un aumento del volumen ventricular a causa de la succión de la sangre de las aurículas [16].

Torrent-Guasp describe al miocardio ventricular como una banda extendida desde la raíz de la arteria pulmonar hasta la raíz de la arteria aorta [9]. En el miocardio canino, se pudo demostrar con una frecuencia de presentación de $100 \%$ de la banda, describiendo en el espacio un helicoide formando dos cavidades, el ventrículo derecho e izquierdo; contradiciendo a su vez a Anderson et al., que proponen un modelo de malla cardiaca en el que los miocitos están dispuestos de una manera longitudinal y radial, con angulaciones que varían con la profundidad miocárdica, y que relaciona esta distribución arquitectónica con su consecuencia funcional [17].

En tal banda pueden ser distinguidas dos lazadas: la basal y la apexiana, cada una de las cuales está dividida en dos segmentos, todos definidos por particularidades morfológicas: el segmento derecho e izquierdo pertenecen a la lazada basal y los segmentos descendentes y ascendentes componen la lazada apexiana [18].

En el presente estudio, se identificaron las dos lazadas en un $100 \%$ de presentación con sus respectivos segmentos, de acuerdo con la descripción de Torrent-Guasp de que el segmento derecho de la lazada basal discurre desde la raíz de la arteria pulmonar y continúa luego con el segmento izquierdo, que conforma la pared libre del ventrículo derecho. Se encontró que tiene una frecuencia de presentación de un $100 \%$. Se observó que rodeaba el ventrículo izquierdo, terminando la disección en la raíz de la aorta, zona donde se termina la primera lazada; este segmento tuvo una frecuencia de presentación de un $100 \%$. Luego se continuó con la lazada apexiana y se identificaron sus dos componentes: segmento descendente y ascendente, con una frecuencia de presentación de un $100 \%$. Estos segmentos corresponden solo al ventrículo izquierdo, así como lo describe Torrent-Guasp.

De acuerdo con la teoría de Torrent-Guasp, el miocardio canino tendría una secuencia de contracción que empezaría por la contracción de la lazada basal, que da lugar a la constitución de una rígida coraza externa que al enfundar y desenfundar la lazada apexiana, mediante un correspondiente descenso (contracción del segmento descendente) y ascenso (contracción del segmento ascendente), origina la expulsión y la succión ventricular. En caninos se han desarrollado trabajos de movimiento rotacional del ápice cardiaco con respecto a su base con resonadores, y como resultado hay una deformación rotacional del ventrículo izquierdo, que es dependiente del patrón de activación del ventrículo izquierdo y el estado contráctil [19].

El mecanismo mediante el cual el corazón lleva a cabo su actividad contráctil, la disminución (expulsión de sangre) y la dilatación (succión de sangre) de ambas cavidades ventriculares, requiere una nueva interpretación, como lo describe Torrent-Guasp. Usualmente, todavía es aceptado que en el miocardio ventricular los estímulos llegan a lo largo del haz de His, atravesando las paredes ventriculares en el sentido endocardio-epicardio. De otro lado, es igualmente admitido que los músculos papilares son las primeras estructuras contráctiles eléctricamente activadas en cada ciclo cardiaco [20].

Posiblemente, la aparición y el mantenimiento de tales creencias sobre la difusión del estímulo por el miocardio ventricular llegan en gran parte condicionados por la carencia, en aquellos tiempos, de un conocimiento coherente sobre la estructura macroscópica del miocardio ventricular. Y aquellas viejas creencias han prevalecido hasta la actualidad, durante casi un siglo, a pesar de la existencia de evidencias experimentales abiertamente opuestas a su validez. Sands-Robb y Robb se preguntaban cómo era posible que la transmisión de los impulsos tenga lugar desde la cara endocárdica a la pericárdica, una indicación solo verificable en la cara anterior del ventrículo izquierdo. Postularon una difusión longitudinal del estímulo a lo largo de los haces musculares, es decir, una difusión axial no en sentido transversal como planteaban los clásicos (indicando que la conducción del proceso de excitación tiene lugar axialmente en la musculatura, a lo largo de una trayectoria paralela a la dirección de la fibra) [21].

En 1979, Armour y Randall evidenciaron, con experimentos en las paredes ventriculares, que la contracción de la musculatura más superficial, la subepicárdica, precede a la contracción de la musculatura profunda, la subendocárdica. Así, quedó demostrado que la interpretación clásica de que la difusión del estímulo tiene lugar en el sentido endocardio-epicardio era errónea. Con nuestro estudio, 
podemos evidenciar que una ruta clara trazada por la banda, al discurrir desde la raíz de la arteria pulmonar hasta la raíz de la aorta, condicionará una progresión longitudinal del estímulo que determina una secuencia en la contracción de las diversas regiones de la banda miocárdica [22].

La contracción del segmento derecho (como es sabido, la estimulación del ventrículo derecho precede a la del ventrículo izquierdo) es seguida por la del segmento izquierdo, hecho confirmado por Armour y Randall al observar la conformación inicial de aquel rígido caparazón externo (expresión de la contracción de la lazada basal que discurre por todo el perímetro de la masa ventricular), en cuyo interior tiene lugar la contracción subsiguiente del resto de la masa miocárdica: la lazada apexiana [5].

Debido a la trayectoria helicoidal de la lazada apexiana, los segmentos descendentes y ascendentes quedan sobrepuestos; por lo tanto, el segmento descendente adopta una situación subendocárdica, mientras el ascendente discurre de manera subepicárdica. El segmento descendente es el que está situado a continuación del segmento izquierdo; por tal motivo, la difusión del estímulo será en la musculatura subendocárdica, representada por el segmento descendente, que precede al inicio de toda actividad eléctrica en la musculatura subepicárdica, presencia que ha sido demostrada por Armour y Randall. Ellos afirman que la actividad eléctrica avanza desde el endocardio hasta el epicardio en la cara anterior de la pared ventricular izquierda. En tal hecho reside lo equívoco de la interpretación de Lewis al generalizar, en todo el corazón, una supuesta difusión endocardio-epicardio del estímulo [23].

Por razones basadas en la estructura macroscópica del miocardio y en los datos experimentales referidos, queda la secuencia representada de la activación eléctrica del miocardio ventricular. Dicha secuencia vendría expresada por la cadena de los sucesivos segmentos de la banda: derecho-izquierdo-descendente-ascendente, donde podemos observar que la activación eléctrica escalonada de los segmentos viene corroborada por las observaciones de Sands-Robb y Robb, quienes señalaban la presencia y el papel dieléctrico de las envolturas conectivas de las fibras miocárdicas. Estas últimas encajan perfectamente con los datos experimentales aportados por Sands-Robb y Robb, según los cuales la difusión de los estímulos tiene lugar a lo largo de los haces musculares, y con los aportados por Armour y Randall, quienes registran la aparición de actividad eléctrica en las diversas regiones de los ventrículos. Con lo anterior, se demuestra la equivocada suposición de los fisiólogos clásicos (Lewis) que interpretaron en las primeras décadas del siglo $\mathrm{xx}$ que el estímulo atraviesa todo obstáculo interpuesto en su camino, al avanzar en sentido endocardio-epicardio atravesando las paredes ventriculares [5].

El mecanismo determinante, según TorrentGuasp, de la expulsión y de la succión ventricular, se desarrolla de la siguiente manera: cuando el corazón se encuentra en reposo, se inicia un nuevo ciclo: primero tiene lugar la contracción del segmento derecho, que es seguida del izquierdo por la relación de continuidad existente entre ambos, y después, tiene lugar la contracción del segmento descendente y del ascendente.

Los resultados de esta contracción de la lazada basal derecha e izquierda producen una reducción del diámetro transversal de los ventrículos (constricción o estrechamiento). Tras la contracción de la lazada basal, sobreviene la contracción de la lazada apexiana; se activa primero la actividad del segmento descendente, que origina un descenso de la base ventricular, dando lugar a un acortamiento de los ventrículos. Inmediatamente, sigue la contracción del segmento ascendente, que al no poder provocar un mayor descenso de la base ventricular, da origen a un envaramiento de sus fibras mediante el cual tiene un ascenso de la base ventricular y simultáneamente un desplazamiento [5].

La unión de los datos morfológicos aportados por la evidencia de la estructuración macroscópica del miocardio ventricular canino, junto con los resultados de diversos trabajos experimentales sobre varias facetas funcionales del corazón y la posibilidad de apreciar directamente la dinámica cardiaca mediante las modernas tecnologías diagnósticas utilizadas en clínica, ha permitido avanzar en una coherente explicación del mecanismo al que recurren los ventrículos para llevar a cabo la expulsión y la succión de sangre.

Este es un nuevo concepto de la anatomía del miocardio ventricular canino, en el que se ha evidenciado macroscópicamente la entramada red de la doble helicoide y se ha demostrado la presencia de cada uno de sus segmentos. Es innegable la necesidad de emprender, en todos los campos de la cardiología, estudios con los cuales precisar detalladamente la funcionalidad del corazón y todas las derivaciones clínicas que este nuevo concepto de la mecánica cardiaca pueda producir a nivel diagnóstico y terapéutico. 


\section{Agradecimientos}

A los doctores Claudia García, Ariel Mendoza y Alejandra Echavarriaga, por sus aportes académicos, su interés y entusiasmo investigativo, y por el suministro del material de investigación, lo cual hizo posible culminarlo con éxitos esperados. Trabajo realizado en memoria del Dr. Francisco Torrent-Guasp.

\section{Referencias}

[1] Budras DK, McCarthy PH, Fricke W, Richter R. Anatomy of the dog: an illustrated text. Múnich: Schlutersche; 2007.

[2] Sisson S, Grossman JD. Sistema vascular: anatomía de los animales domésticos. Barcelona: Salvat Editores; 2000.

[3] Randall D, Burggren W, French K. Circulación: fisiología animal, mecanismos y adaptaciones. Madrid: McGraw-Hill; 1998.

[4] Cunningham G. El corazón como bomba: fisiología veterinaria (3. a ed.). Madrid: Elsevier; 2009.

[5] Torrent-Guasp, F. La estructuración macroscópica del miocardio ventricular. Rev Esp Cardiol. 1980;33:265-87.

[6] Brecher GA. Experimental evidence of ventricular diastolic function. Circ Rev. 1956;4:513-8.

[7] Brecher GA. Critical review of recent works on ventricular diastolic suction. Circ Rev. 1958;6:554-66.

[8] Rivero BM, Gutiérrez RM, Sarasquete MC, Gonzáles CML, García BA. Activación eléctrica cardiovascular, ¿longitudinal y continua? La circulación eléctrica. Rev Esp Cardiol. 1995;1-9.

[9] Torrent-Guasp F. Estructura y función del corazón. Rev Esp Cardiol. 1998;51:91-102.

[10] Poveda F, Gil D, Martí E, Andaluz A, Balester M, et al. Estudio tractográfico de la anatomía helicoidal del miocardio ventricular mediante resonancia magnética por tensor de difusión. Rev Esp Cardiol. 2013;66(10):782-90.

[11] Buckberg GD. Basic science review: the helix and the heart. J Thorac Cardiovasc Surg. 2002;124(5):863-83.

[12] Vázquez F. La anatomía en la Grecia clásica: el periodo alejandrino. Revista del Hospital J.M. Ramos Mejía. 2006;11(1).
[13] Pettigrew JB. On the arrangement of the muscular fibres in the ventricles of the vertebrate heart, with physiological remarks. Phylos Trans. 1864;154:445-500.

[14] Torrent- Guasp F, Kocicab JM, Cornoc A, Komedad MJ, Flotatsf A, et al. Systolic ventricular filling. Eur J Cardiothorac Surg. 2004;25(6):376-86.

[15] Cosín AJ, Martínez HA, Tuzón ST, Agüero JR, Torrent-Guasp F. Estudio experimental de la llamada fase de relajación isovolumétrica del ventrículo izquierdo. Rev Esp Cardiol. 2009;62(4):392-9.

[16] Buchalter MB, Rademakers FE, Weiss JL, Rogers WJ, Weisfeld ML, Shapiro EP. Rotational deformation of the canine left ventricle measured by magnetic resonance tagging: effects of catecholamines, ischaemia and pacing. Cardiovasc Res. 1994;28(5):629-35.

[17] Anderson RH, Ho SY, Redmann K, Sanchez-Quintana D, Lunkenheimer PP. The anatomical arrangement of the myocardial cells making up ventricular mass. Eur J Cardiothorac Surg. 2005;28(4):517-25.

[18] Cosín J, Hernándiz A. La disposición de las fibras miocárdicas en una banda condiciona la morfología y la función del corazón. Rev Esp Cardio. 2013;66(10):768-70.

[19] Badke FR, Boinay P, Covell JW. Effects of ventricular pacing on regional left ventricular performance in the dog. Arts T Physiol. 1980;238(6):H858-67.

[20] Trainini JC, Hereros J. ¿El corazón es una bomba de succión? Agonista Rev Arg Cardiol. 2011;79(1).

[21] Torrent-Guasp F, Ballester MD, Buckber GD, Carreras F, Floats A, et al. Spatial orientation of the ventricular muscle band: physiologic conribution and surgical implications. J Thorac Cardiovasc Surg. 2011;122(2):389-92.

[22] Kocica MJ, Corno AF, Carreras-Costa F, Ballester-Rodes M, Moghbel MC, Cueva CN, et al. The helical ventricular myocardial band: global, three-dimensional, functional architecture of the ventricular myocardium. Eur J Cardiothorac Surg. 2006;29(Suppl 1):S21-S40.

[23] Torrent-Guasp F. La mecánica agonista-antagonista de los segmentos descendente y ascendente de la banda miocárdica ventricular. Rev Esp Cardiol. 2001;54(9):1091-102. 